\title{
A NOTE ON PARTS AND HYPERBOLIC GEOMETRY
}

\section{JOSEPH LEWITTES ${ }^{1}$}

1. Let $A$ be a normed linear space over the field $K$, where $K$ is either the real field $R$ or complex field $C ; \Delta(A)=\{a \in A \mid\|a\|<1\}$, $\Sigma(A)=\{a \in A|| \mid a \|=1\}, \Delta=\{z \in C|| z \mid<1\}$. The hyperbolic metric on $\Delta$ is

$$
\rho(x, y)=\frac{1}{2} \log \frac{1+[x, y]}{1-[x, y]}, \quad \text { where } \quad[x, y]=\left|\frac{x-y}{1-x \bar{y}}\right| ;
$$

see $\left[1\right.$, p. 238] for details. Define the relation $\sim$ on $\Delta\left(A^{*}\right) \cup \Sigma\left(A^{*}\right)$, $A^{*}$ the dual space of $A$, by $L_{1} \sim L_{2}$ if and only if $\sup _{a \in \Delta(A)} \rho\left(L_{1}(a)\right.$, $\left.L_{2}(a)\right)<\infty$. As $\rho$ is a metric, $\sim$ is an equivalence relation. The equivalence classes under $\sim$ will be called hyperbolic parts. It is easily seen that $\Delta\left(A^{*}\right)$ is a single hyperbolic part; hence the interest is in the decomposition of $\Sigma\left(A^{*}\right)$.

2. Before stating our theorems, we recall that an open segment $\sigma$ in $A^{*}$ is a set of the form $\sigma=\left\{t L_{1}+(1-t) L_{2}\right\}_{0<t<1}, L_{1}, L_{2} \in A^{*}$. If $K=C$, an analytic disk, $\delta$ in $A^{*}$ is a set of the form $\delta=f(\Delta)$, where $f: \Delta \rightarrow A^{*}$ is analytic. For our purposes a map $f: \Delta \rightarrow A^{*}$ is analytic if for every $a \in A$ the complex valued function $z \rightarrow L_{z}(a)$ is analytic, where $L_{z}=f(z)$.

Theorem 1. Let $L_{1}, L_{2} \in \Sigma\left(A^{*}\right)$ and belong to the same hyperbolic part $P$. (a) There is an open segment $\sigma$ such that $L_{1} \in \sigma, L_{2} \in \sigma$ and $\sigma \subset P$. (b) If $K=C$ there is an analytic disk $\delta$ such that $L_{1} \in \delta, L_{2} \in \delta$ and $\delta \subset P$.

The proof depends on an inequality which is interesting in its own right. Let $I$ denote the unit interval $0 \leqq t \leqq 1$ and for $\epsilon>0, I_{\epsilon}$ $=\{z \in C|| z-t \mid<\epsilon$ for some $t \in I\}$.

Lemma. Given $0<k<k+\theta<1$ and $\epsilon=\theta / k(1+k+\theta)$. If $x, y \in \Delta$, $[x, y] \leqq k$, then for $z \in I_{\epsilon}, y+z(x-y) \in \Delta$ and $[y+z(x-y), x] \leqq k+\theta$.

Proof. If $a \in \Delta, b \in C$, then $[a, b]<1$ if and only if $b \in \Delta$; hence it

Received by the editors January 14, 1966.

${ }^{1}$ Research done at Harvard University. A first draft was written at Harvard with the support of the U. S. Army Research Office (Durham). This paper was revised at Yeshiva University with the Support of National Science Foundation Grant GP-5315. 
suffices to prove only that $[y+z(x-y), x] \leqq k+\theta$. It is easily verified that our hypotheses imply that for $t \in I$,

$$
0<\frac{1+\epsilon-t}{1 / k-\epsilon-t} \leqq \frac{1+\epsilon}{1 / k-\epsilon}=k+\theta .
$$

Given $z \in I_{\epsilon}$ choose $t \in I$ such that $|z-t|<\epsilon$, so that $|1-z| \leqq 1+\epsilon-t$ and $|z| \leqq \epsilon+t$. Then

$$
\begin{aligned}
{[y+z(x-y), x] } & =\left|\frac{1-z}{(1-y \bar{x}) /(y-x)+z \bar{x}}\right| \\
& \leqq \frac{1+\epsilon-t}{|1 /[y, x]-| z|| \bar{x}||} \leqq \frac{1+\epsilon-t}{1 / k-\epsilon-t} \leqq k+\theta,
\end{aligned}
$$

which proves the lemma.

Proof of Theorem 1. $L_{1} \sim L_{2}$ implies $\sup _{a \in \Delta(A)}\left[L_{1}(a), L_{2}(a)\right]$ $=k<1$ so that if $k, \theta, \epsilon$ are as in the lemma, the open segment $\sigma=\left\{L_{(z)}=L_{1}+z\left(L_{2}-L_{1}\right),-\epsilon<z<1+\epsilon\right\}$ is contained in $P$. Indeed, by the lemma, $\left\|L_{(z)}\right\|=\sup _{a \in \Delta(A)}\left|L_{1}(a)+z\left(L_{2}(a)-L_{1}(a)\right)\right| \leqq 1$ and $\sup _{a \in \Delta(A)}\left[L_{(z)}(a), L_{2}(a)\right] \leqq k+\theta$ so that $L_{(z)} \sim L_{2}$ for every $z$, hence $\sigma \subset P$. Also $L_{1}=L_{(1)} \in \sigma, L_{2}=L_{(1)} \in \sigma$. If $K=C$, choose a conformal map $f$ of $\Delta$ onto $I_{\epsilon}$, a simply connected domain, say $z=f(\zeta)$; it again follows from the lemma that the analytic disk $\delta=\left\{L_{(\zeta)}=L_{1}+f(\zeta)\left(L_{2}\right.\right.$ $\left.\left.-L_{1}\right), \zeta \in \Delta\right\}$ is contained in $P$ and $L_{1}=L_{\left(\gamma^{-1}(0)\right)} \in \delta L_{2}=L_{\left(\gamma^{-1}(1)\right)} \in \delta$. q.e.d.

The next theorem investigates the converse of the first.

TheOREM 2. Let $P$ be a hyperbolic part of $\Sigma\left(A^{*}\right)$. (a) If $K=R, \sigma$ an open segment $\subset \Sigma\left(A^{*}\right) \cup \Delta\left(A^{*}\right)$ and $\sigma \cap P \neq \varnothing$, then $\sigma \subset P$.

(b) If $K=C, \delta$ an analytic disk $\subset \Sigma\left(A^{*}\right) \cup \Delta\left(A^{*}\right)$ and $\delta \cap P \neq \varnothing$, then $\delta \subset P$.

(c) If $K=C$,(a) is false.

Proof. (a) If (a) is false there is an open segment $\sigma \subset \Sigma\left(A^{*}\right) \cup \Delta\left(A^{*}\right)$ and $L_{1}, L_{2} \in \sigma$ such that $L_{1} \in \sigma \cap P$ and $L_{2} \nsim L_{1}$. Thus we can find a sequence $a_{n} \in \Delta(A)$ such that if $x_{n}=L_{1}\left(a_{n}\right), y_{n}=L_{2}\left(a_{n}\right)$, then $x_{n} \rightarrow 1$, $y_{n} \rightarrow \alpha,-1 \leqq \alpha \leqq 1$, and $\left[x_{n}, y_{n}\right] \rightarrow 1$ as $n \rightarrow \infty$. Since $\sigma$ is open, for all sufficiently small $\epsilon>0,\left\|L_{1}+\epsilon\left(L_{1}-L_{2}\right)\right\| \leqq 1$ and in particular, $\left|x_{n}+\epsilon\left(x_{n}-y_{n}\right)\right|<1$ for all $n$. Clearly this last inequality is impossible for all $n$ if $\alpha<1$. If $\alpha=1$ we may assume that $x_{n}>y_{n}$ for all $n$, for otherwise we can interchange $L_{1}$ and $L_{2} .\left|x_{n}+\epsilon\left(x_{n}-y_{n}\right)\right|<1$ implies

$$
1>\left[y_{n}, x_{n}+\epsilon\left(x_{n}-y_{n}\right)\right]=\frac{1+\epsilon}{\left|\left(1-y_{n} x_{n}\right) /\left(x_{n}-y_{n}\right)-\epsilon y_{n}\right|} .
$$


As $n \rightarrow \infty$ this last expression tends to $(1+\epsilon) /(1-\epsilon)>1$. A contradiction.

(b) Recall Pick's formulation of Schwarz's lemma: [1, p. 239]. If $f: \Delta \rightarrow \Delta$ is analytic then for $z_{1}, z_{2} \in \Delta, \rho\left(f\left(z_{1}\right), f\left(z_{2}\right)\right) \leqq \rho\left(z_{1}, z_{2}\right)$. Given an analytic disk $\delta=\left\{L_{z}, z \in \Delta\right\}$, since $\left\|L_{z}\right\| \leqq 1$ by hypothesis, for each $a \in \Delta(A), z \rightarrow L_{z}(a)$ is an analytic map of $\Delta \rightarrow \Delta$. Thus if $L_{z_{0}} \in \delta \cap P$, $L_{z} \in \delta$ we have $\sup _{a \in \Delta(A)} \rho\left(L_{z_{0}}(a), L_{z}(a)\right) \leqq \rho\left(z_{0}, z\right)<\infty$, hence $L_{z} \sim L_{z_{0}}$ and $\delta \subset P$.

(c) Let $A$ be the complex Banach space of sequences of complex numbers $a=\left(\alpha_{1}, \alpha_{2}, \cdots\right)$ with $\|a\|=\sum_{n=1}^{\infty}\left|\alpha_{n}\right|<\infty$. Then $A^{*}$ is the space of sequences of complex numbers $L=\left(\lambda_{1}, \lambda_{2}, \cdots\right)$ with $\|L\|$ $=\sup _{n}\left|\lambda_{n}\right|<\infty, L(a)=\sum_{n=1}^{\infty} \lambda_{n} \alpha_{n}$. We first note that if $L_{1}=\left(x_{n}\right)$, $0 \leqq x_{n}<1$ for all $n, x_{n} \rightarrow 1$ and $L_{2}=\left(x_{n}+i y_{n}\right), y_{n}$ real, $x_{n}^{2}+y_{n}^{2}<1, y_{n} \rightarrow 0$ then $L_{1}, L_{2} \in \Sigma\left(A^{*}\right)$ and $L_{1} x L_{2}$ if $\lim \sup _{n \rightarrow \infty}\left(\left|y_{n}\right| /\left(1-x_{n}\right)\right)=\infty$, i.e., $x_{n}+i y_{n} \rightarrow 1$ "tangentially." For, if $\delta_{n} \in A$ has 1 in the $n$th place and 0 otherwise, $\left\|t \delta_{r}\right\|<1$ for any $|t|<1$ and

$$
\sup _{a \in \Delta(A)} \rho\left(L_{1}(a), L_{2}(a)\right) \geqq \sup _{n,|t|<1} \rho\left(L_{1}\left(t \delta_{n}\right), L_{2}\left(t \delta_{n}\right)\right) .
$$

But

$$
\left[L_{1}\left(t \delta_{n}\right), L_{2}\left(t \delta_{n}\right)\right]=\frac{1}{\left|\left(1-t x_{n}\right)\left(1+t x_{n}\right) / t y_{n}+i t x_{n}\right|}
$$

which can be made arbitrarily close to 1 by choosing $n$ sufficiently large and $t$ close to 1 . Thus $\sup _{a \in \Delta(A)} \rho\left(L_{1}(a), L_{2}(a)\right)=\infty$ and $L_{1} \nsim L_{2}$. But such $L_{1}, L_{2}$ may lie on an open segment in $\Sigma\left(A^{*}\right)$; as a concrete example consider $L_{1}=\left(x_{n}\right), x_{n}=1-1 / n$ for $n \geqq 2$ and $x_{1}=0, L_{2}$ $=\left(x_{n}+i y_{n}\right)$ where $y_{n}=n^{-1 / 2}$ for $n \geqq 2$ and $y_{1}=0$. Then $\left\|L_{1}\right\|=\left\|L_{2}\right\|=1$ and since $y_{n} /\left(1-x_{n}\right)=n^{1 / 2} \rightarrow \infty, L_{1} \nsim L_{2}$. But the open segment $\sigma=\left\{L_{1}+t\left(L_{2}-L_{1}\right) ;-\epsilon<t<1+\epsilon\right\}$ is contained in $\Sigma\left(A^{*}\right)$ for suitably small $\epsilon>0$. For $L_{1}+t\left(L_{2}-L_{1}\right)=\left(x_{n}+i t y_{n}\right)$ and

$$
\left|x_{n}+i t y_{n}\right|^{2}=1+\frac{1}{n^{2}}+\frac{t^{2}-2}{n} \leqq 1+\frac{1}{n^{2}}+\frac{\epsilon^{2}+2 \epsilon-1}{n} \leqq 1
$$

for all $n>1$ if $\epsilon^{2}+2 \epsilon \leqq \frac{1}{2}$. q.e.d.

3. As a consequence of Theorem 1 (a) we have that if $L_{1}, L_{2}$ $\in \Sigma\left(A^{*}\right)$ are in the same hyperbolic part, then $\left\|L_{1}-L_{2}\right\|<2$. In fact since they are on an open segment $\sigma \subset \Sigma\left(A^{*}\right)$, for suitably small $\epsilon>0, \Sigma\left(A^{*}\right)$ contains $L_{2}+\epsilon\left(L_{2}-L_{1}\right)$ and $L_{1}+\epsilon\left(L_{1}-L_{2}\right)$ so that $2 \geqq(1+2 \epsilon)\left\|L_{1}-L_{2}\right\|$. On the other hand, the example of Theorem 2 (c) shows that $\left\|L_{1}-L_{2}\right\|<2$ does not imply that $L_{1}$ and $L_{2}$ are in the same hyperbolic part. The idea of introducing the hyperbolic metric 
to functional analysis to obtain an equivalence relation is due to A. Gleason. In his original paper [2] this however is not made explicit. In the context of [2], $A$ is a function algebra, $L_{1}, L_{2}$ are homomorphisms of $A$ and the equivalence relation is defined as $L_{1} \sim L_{2}$ if $\left\|L_{1}-L_{2}\right\|<2$. In this case this does imply $\rho\left(L_{1}, L_{2}\right)<\infty$; for, $A$ being a function algebra, if $T(\zeta)=\lambda(\zeta-\mu) /(1-\bar{\mu} \zeta),|\lambda|=1,|\mu|<1$ is any conformal automorphism of $\Delta$, and $a \in \Delta(A)$, there is a unique $\hat{T}(a)$ $\in \Delta(A)$ such that for any homomorphism $L$ of $A, T(L(a))=L(\hat{T}(a))$. Furthermore, given $\zeta_{i}, w_{i} \in \Delta(i=1,2)$ and $\rho\left(\zeta_{1}, \zeta_{2}\right)=\rho\left(w_{1}, w_{2}\right)$ there is an automorphism $T$ of $\Delta$ with $T\left(\zeta_{i}\right)=w_{i}(i=1,2)$, in particular we can find $r, 0 \leqq r<1$ such that $\rho\left(\zeta_{1}, \zeta_{2}\right)=\rho(r,-r)$. Thus, if $L_{1}, L_{2}$ are homomorphisms of $A, \rho\left(L_{1}, L_{2}\right)=\sup \rho(r,-r)$, the supremum taken over all $a \in \Delta(A)$ with $L_{1}(a)=r, L_{2}(a)=-r$. Hence $\left\|L_{1}-L_{2}\right\|$ $\geqq \sup 2 r=2$ if $\rho\left(L_{1}, L_{2}\right)=\infty$. Another notion of part has been introduced by Bear [3] for $A$ a real linear space of real continuous functions on a compact Hausdorff space $X$. The essential property of these parts is that they are characterized by conditions (a) of Theorem 1 and 2. Thus the concept of hyperbolic part introduced here coincides with previous definitions of "part" where applicable.

In conclusion, I would like to thank Professor A. Gleason for several helpful discussions concerning parts and hyperbolic geometry.

\section{BIBLIOGRAPHY}

1. E. Hille, Analytic function theory, Vol. II, Ginn, Boston, Mass., 1962.

2. A. Gleason, Function algebras, Seminars on Analytic Functions, Vol. 2, Princeton Univ. Press, Princeton, N. J., 1957.

3. H. S. Bear, A geometric characterization of Gleason parts, Proc. Amer. Math. Soc. 16 (1965), 407-412.

Yeshiva UNIVERSITY 